REVIEW ARTICLE

\title{
Two-dimensional Tracking Technique for Assessment of Fetal Cardiac Function
}

\author{
Sumito Nagasaki ${ }^{1}$, Masahiko Nakata ${ }^{2}$
}

\begin{abstract}
Although various methods have been reported to evaluate fetal cardiac function using ultrasound, two-dimensional speckle-tracking echocardiography (2D-STE), which automatically tracks speckles on B-mode images has the advantage of being angle-independent. Several ultrasound devices are now capable of evaluating fetal cardiac function using 2D-STE, like global longitudinal strain (GLS), wall strain, and fractional area change (FAC). There is also a method called auto fractional shortening (FS) that can automatically calculate the fractional shortening (FS). The most important thing for 2D-STE measurement is to make the B-mode image clear. So, it is important to display the heart as large as possible and to use the highest frame rate possible. There have been many reports of normal and pathological fetuses. However, there are some problems, such as the reference value varies depending on the device or algorithm, and the measurement can only with highend ultrasound devices, so further development is expected.
\end{abstract}

Keywords: Auto FS, Fetal cardiac function, Strain, Two-dimensional speckle-tracking echocardiography.

Donald School Journal of Ultrasound in Obstetrics and Gynecology (2021): 10.5005/jp-journals-10009-1707

\section{INTRODUCTION}

Assessment of fetal cardiac function with echocardiography is performed by various methods such as the B-mode, M-mode, pulseDoppler, tissue Doppler, and tracking techniques. ${ }^{1}$ From among these, the evaluation of the ventricular wall movement is often reported by the $\mathrm{M}$-mode or the tissue Doppler method. However, measurement using M-mode and tissue Doppler are limited to an angle formed by the beam and movement of the tissue. On the other hand, the two-dimensional tracking (2DT) technique which uses a B-mode image is angle-independent. As such, recently there have been many reports of fetal cardiac function measurements using the 2DT technique. Currently, the 2DT technique often refers to two-dimensional speckle-tracking echocardiography (2D-STE).

\section{Principle of 2D Speckle-tracking Echocardiography}

Two-dimensional speckle-tracking echocardiography automatically tracks high-intensity point-like speckles (images created by the interference of ultrasonic waves backscattered by structures smaller than the wavelength of ultrasonic waves). A small area including this speckle is compared with frame $n$ and the next frame $n+1$ in the search field. The place with the smallest error in the search field is detected and followed as the destination of the movement (Fig. 1). This is done in continuous frames (Fig. 2), and the moving distance and moving speed of the region of interest (ROI) are calculated accordingly. The biggest advantage is that by using the $B$-mode image as described above, it is possible to track in all directions with less influence of the angle dependence, which is a problem with the Doppler method. ${ }^{2-4}$ However, given the principle of tracking the speckle, the analysis depends on the quality and frame rate of the B-mode image.

\section{Ultrasound Devices}

Currently, there are ultrasound devices such as the Artida (Toshiba Medical Systems; Tokyo, Japan), ACUSON (Siemens Healthineers Global; CA, USA), Vivid, and Voluson (General Electronics Healthcare; Milwaukee, USA) that can measure fetal cardiac functioning using 2D-STE. In addition, each ultrasound device uses a variety of \begin{tabular}{l}
\hline \hline${ }^{1,2}$ Department of Obstetrics and Gynecology, Toho University Omori \\
Medical Center, Omori-nishi, Ota-ku, Tokyo, Japan \\
Corresponding Author: Sumito Nagasaki, Department of Obstetrics \\
and Gynecology, Toho University Omori Medical Center, Omori- \\
nishi, Ota-ku, Tokyo, Japan, Phone: +03-3762-4152, e-mail: sumito. \\
nagasaki@med.toho-u.ac.jp \\
How to cite this article: Nagasaki S, Nakata M. Two-dimensional \\
Tracking Technique for Assessment of Fetal Cardiac Function. Donald \\
School J Ultrasound Obstet Gynecol 2021;15(3):254-258. \\
Source of support: Nil \\
Conflict of interest: None
\end{tabular}

software including Vector Velocity Imaging (VVI) (Siemens), STE Echo PAC (Tom Tec; Munich, Germany), Automated Functional Imaging (AFI) Echo PAC software (GE), and Wall Motion Tracer (WMT) (Toshiba).

\section{What can be Measured by the 2D-STE METHOD?}

\section{Global Strain}

Figure 3 shows an example of what can be measured by the 2D-STE method in the fetal heart. From among them, the global longitudinal strain (GLS), a strain in the long axis direction in the entire ventricle, is the most common in the fetal heart. The name of the strain differs depending on the contraction direction of the myocardium. Other strains include the radial and circumferential strains (Fig. 4). The radial strain shows a positive value, and the circular and longitudinal strains are calculated as negative values because they are shortened by contraction. The main reason for evaluation by tracking the entire ventricle in the fetus is that it is unlikely that partial functional deterioration such as coronary artery lesions in adults will occur in the fetus. Although there have been many reports on $\mathrm{GLS}^{5-11}$ van Oostrum et al. concluded in their systematic review that it is difficult to provide a reference

(c) Jaypee Brothers Medical Publishers. 2021 Open Access This article is distributed under the terms of the Creative Commons Attribution 4.0 International License (https://creativecommons.org/licenses/by-nc/4.0/), which permits unrestricted use, distribution, and non-commercial reproduction in any medium, provided you give appropriate credit to the original author(s) and the source, provide a link to the Creative Commons license, and indicate if changes were made. The Creative Commons Public Domain Dedication waiver (http://creativecommons.org/publicdomain/zero/1.0/) applies to the data made available in this article, unless otherwise stated. 

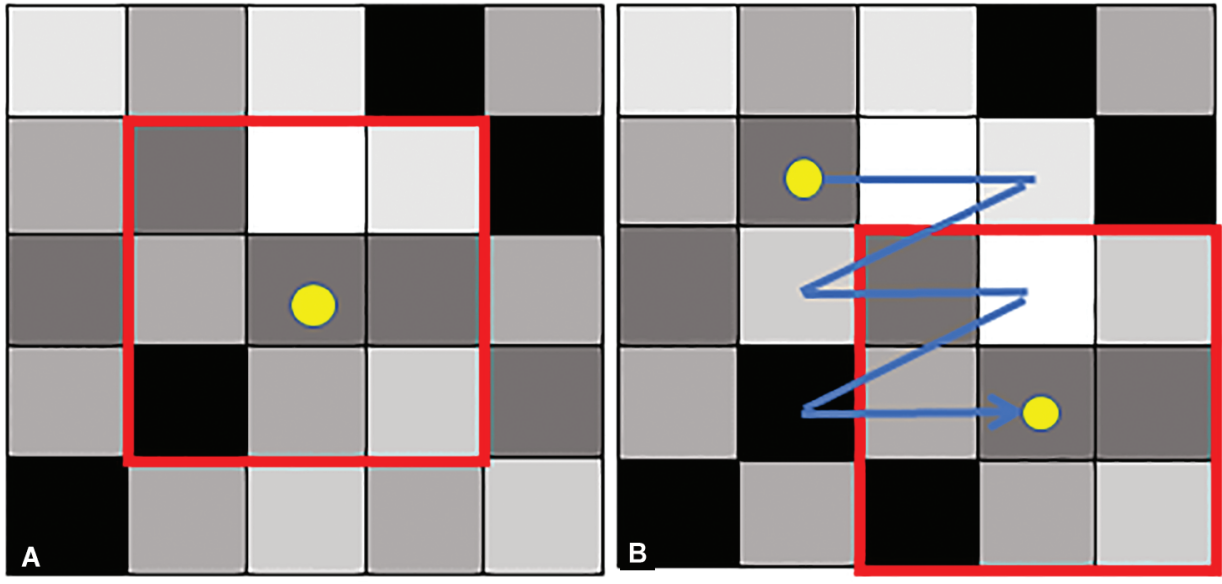

Figs $1 \mathrm{~A}$ and $\mathrm{B}$ : (A) A collection of speckles in $\mathrm{n}$ frame. An area (red framed) centered by region of interest (ROI) (yellow dot) set by the examiner is used as the template; (B) A collection of speckles in the $n+1$ frame. The entire area of $\mathrm{b}$ is calculated and compared. The red-framed area in the bottom right with the largest overlap is tracked as the movement destination

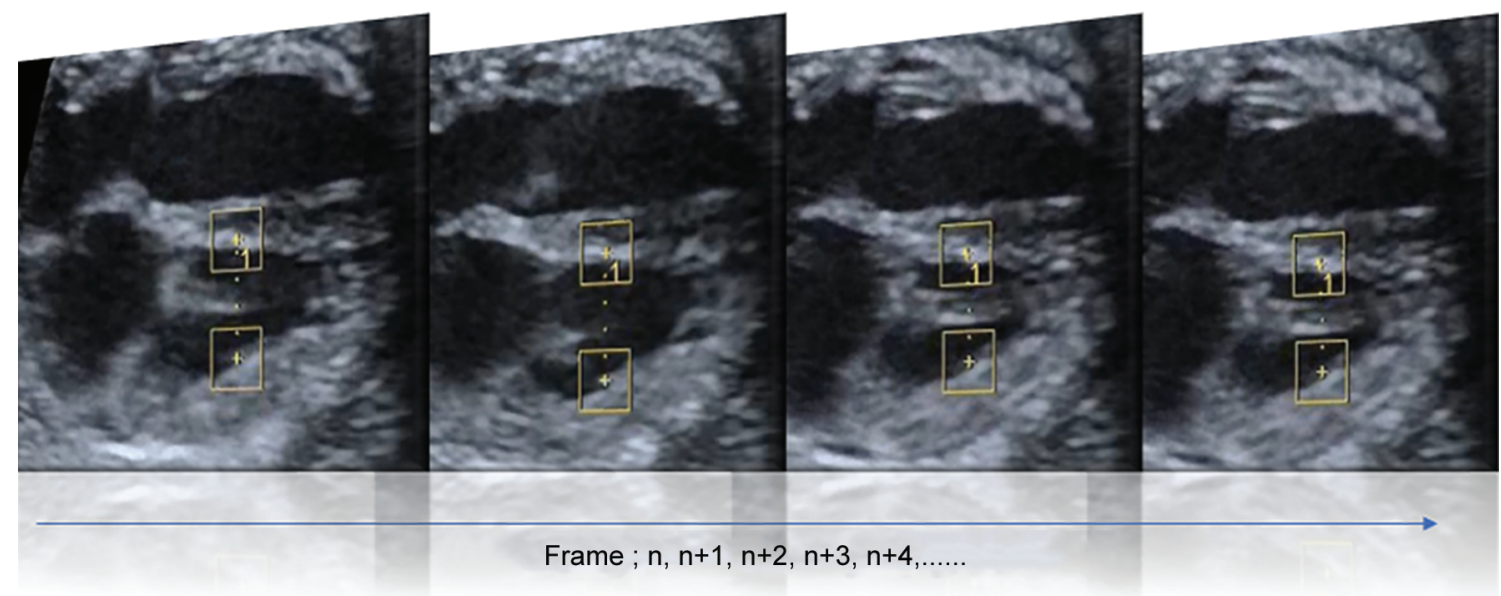

Fig. 2: The region of interest (ROI) set checks and tracks the movement of the target per frame
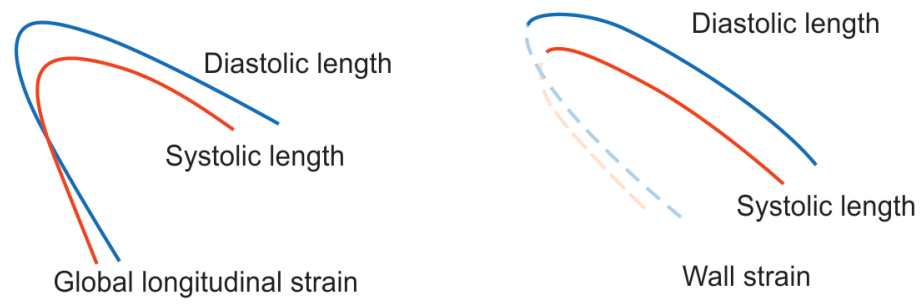

Wall strain
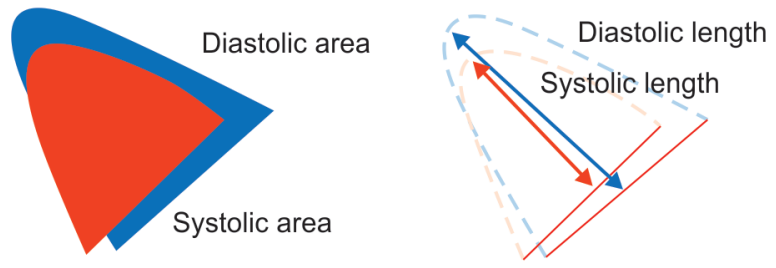

Fractional area change Basal-apical fractional shortening

Fig. 3: An example of the measurement of ventricular function obtained using 2D-STE

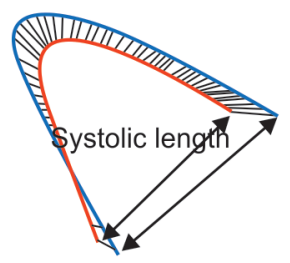

24-segment

transverse fractional shortening 


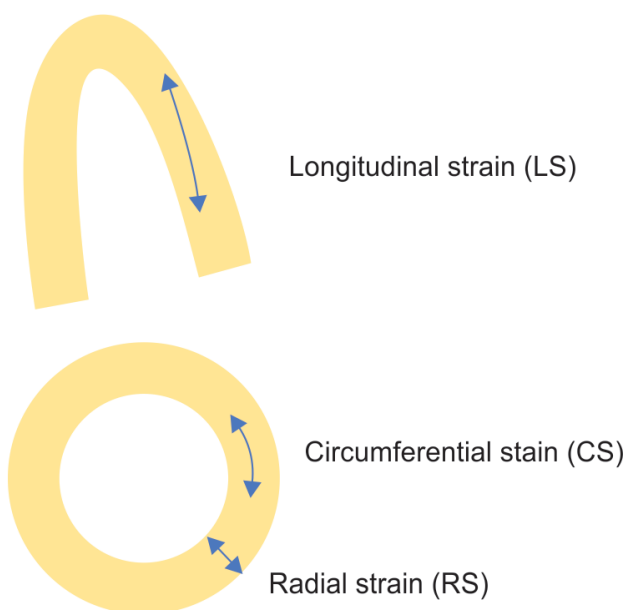

Fig. 4: Types of strains classified in terms of the direction of myocardial contraction

value during pregnancy. ${ }^{2}$ This is because when 23 papers were reviewed in the systematic review, GLS was reported from any tendency of "increase", "decrease", or "constancy" in proportion to the number of weeks of pregnancy. About ultrasound device, in the systematic review, nine measuring devices from Siemens, nine from GE (vivid $\times 6$, volson $\times 3$ ), two from Toshiba, and three from two or more devices. In terms of software, 15 were VVI, 3 were speckle-tracking software provided by TomTec, 2 were STE Echo PAC, 2 were WMT software, and 1 was AFI Echo PAC software. As such, Noortje suggested that difference of the GLS may have been due to differences in ultrasound devices and software. However, they also noted that it is unclear whether this contradictory conclusion was due to the research design or the difference in the measurement algorithm. At the very least, we should understand that the reference values vary depending on the device, software, and algorithm used for measurement. Depending on the device, the reference value may be tied to the ultrasound device, which may be useful. Regarding GLS, there have been reports published on abnormal values, and these are described below.

\section{FGR}

FGR due to placental insufficiency is known to be associated with adaptive changes in fetal hemodynamics. In general, changes in cardiac function and cardiac morphology in FGR are considered to occur before fetal growth restriction becomes apparent. ${ }^{12}$ Early cardiac remodeling and decreased cardiac function are apparent in the ductus venosus pulsatility index, fetal heart rate, and Tei index (Myocardial Performance Index: $\mathrm{MPI}){ }^{13}$ but recent evaluation using the tissue Doppler and 2D-STE methods is considered to have good sensitivity to early phase mild myocardial infarction. However, the systematic review ${ }^{14}$ that examined longitudinal strain, strain rate, and dyssynchrony for FGR fetuses by Noortje et al. concluded that, due to changes in strain itself during pregnancy, effects that ultrasound device, software have on values, and differences in the criteria for FGR fetuses for each study, there is no tendency for FGR fetuses. Further prospective cohort studies for FGR are necessary.

\section{Twin-twin Transfusion Syndrome}

Twin-twin transfusion syndrome (TTTS) is a condition through the anastomotic blood vessels on the placenta that develops in about $10 \%$ of monochorionic diamniotic (MD) twins. In general, the recipient twin develops cardiac dysfunction due to hypertension from increased circulatory blood flow and activation of the reninangiotensin-aldosterone system. Although reports ${ }^{15,16}$ have been made using ultrasound parameters so far, evaluation by Doppler such as the Tei index has been the main method. In recent years, it has been reported that the movement of the myocardium using 2D-STE. However, according to Taylor et al., ${ }^{17}$ compared with the donor twin, the recipient twin shows decreased left ventricular strain in Quintero stages I and II, and decreased right ventricular strain in stages III and IV. Rychik et al. ${ }^{18}$ reported that as TTTS worsened, the strain in the right ventricle decreased, ${ }^{15}$ consistent with Wohlmuthn's report. As such, this likely reflects the pathophysiology of TTTS. In addition, Harbison et al. ${ }^{19}$ conducted a retrospective study of the size, shape, and function of the ventricle of recipient twin before and after fetoscopic surgery in 15 TTTS cases. Improvement was reported for many cardiac function parameters such as strain and fractional area change (FAC) in the left ventricle. As such, it can likely be used for postoperative management.

In addition, there have been some reports of fetal mothers with gestational diabetes mellitus, ${ }^{20}$ congenital diaphragmatic hernia, ${ }^{21}$ and congenital heart disease. ${ }^{22}$ Furthermore, there is a report ${ }^{3}$ that strain not only for the ventricles but also for the atria is negatively correlated with the number of weeks of gestation. Two-dimensional speckle-tracking echocardiography is also characterized by the fact that it can record measurements at any site.

\section{Auto FS}

Global longitudinal strain mainly evaluates the entire ventricle. However, auto FS is a technique that can automatically measure the ventricular diameter shortening rate by tracking two locations near the FS measurement position by the conventional M-mode method $^{23,24}$ (Figs 5 and 6). The strength of auto FS is that by using the 2D-STE method, the myocardium contracting toward the cardiac apex is automatically tracked by setting two ROls at once. Unlike the conventional M-mode method, the contraction in the vertical direction is taken into account, and it is possible to evaluate more easily and accurately. In fact, both the intra- and interobsever correlation coefficients are high. As a measured value, auto FS has a certain tendency to show a significantly negative correlation with the number of weeks of gestation. The aspects that the authors believe make auto FS superior to the recently reported evaluation method of the entire cardiac intima using 2D-STE are described below. First, it is possible to evaluate even when the entire circumference of the endocardium cannot be followed. Second, the current 2D-STE-based evaluation tool for the entire endocardium is done by the manual setting of one cardiac cycle by the examiner and the measurement is done in that one cardiac cycle. As such, it is only evaluated for one cardiac cycle. On the other hand, in auto FS, the average value of the contraction rate for several cycles can be automatically measured as long as the ROI is set at two points.

\section{IMPORTANT THINGS tO CONSIDER IN THE Evaluation OF 2D-STE}

The most important of measuring using 2D-STE is improving the quality of recorded B-mode images. There are two requirements to obtain a better image. One is to display a four-chamber crosssection image on the entire screen, and the other is to record at a high frame rate. A frame rate of 80 frames per second $(80 \mathrm{~Hz})$ or higher is recommended, depending on the fetal heart rate 

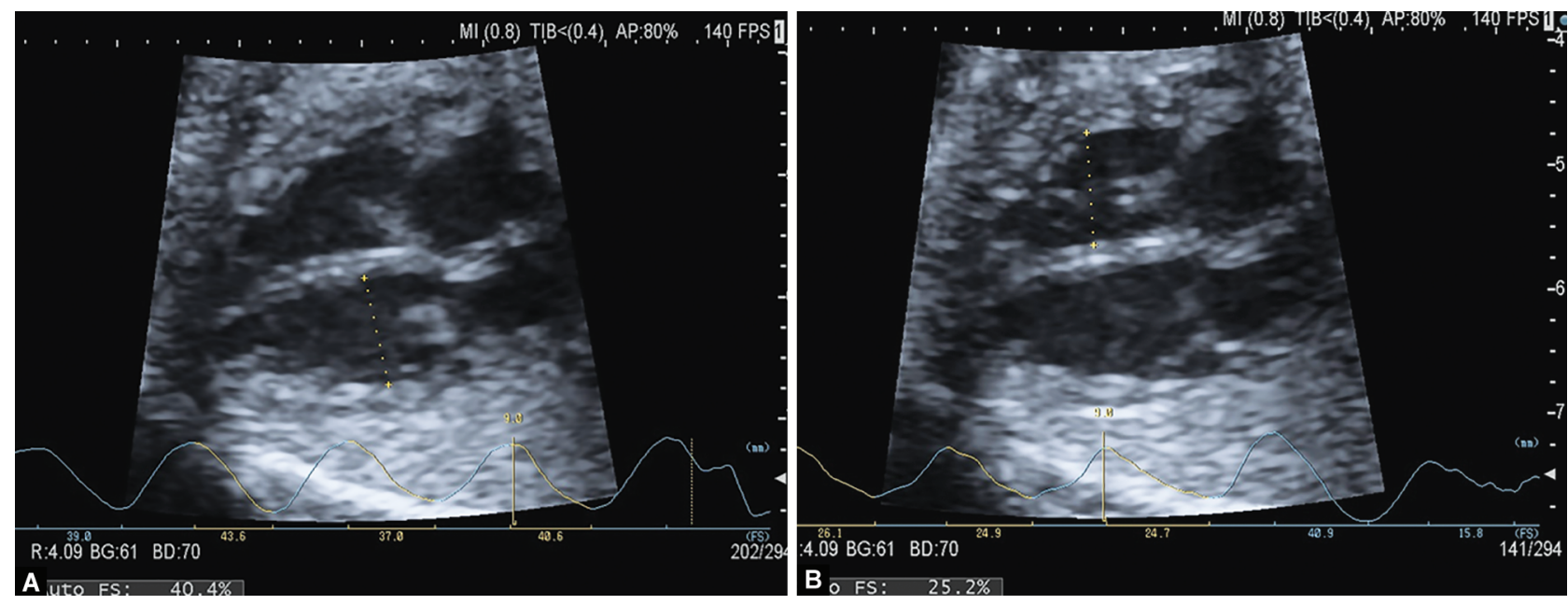

Figs 5A and B: (A) Ultrasonography showing R-auto FS; (B) Ultrasonography showing L-auto FS

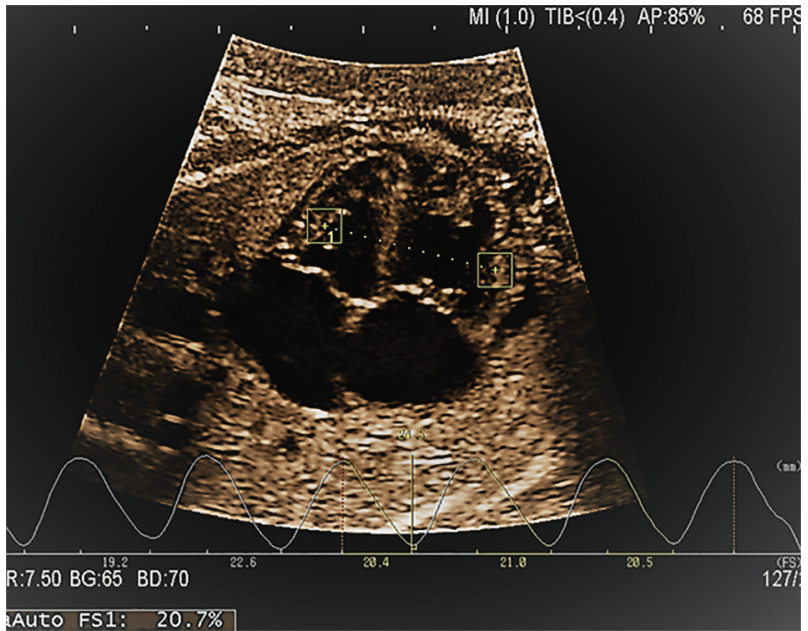

Fig. 6: Combined-auto FS. Measurements with the fetal heart apex facing toward the ventral side of the mother

per minute. ${ }^{25}$ To obtain an image of 40 frames per heart rate, a fetal heart rate of 120 beats/minute and a frame rate of $80 \mathrm{~Hz}$ are required; for a fetal heart rate of 140 beats/minute, a frame rate of $93 \mathrm{~Hz}$ is required.

\section{SUMMARY}

Unlike Doppler evaluation that evaluates blood flow, 2D-STE can evaluate the heart itself because it tracks the myocardium and endocardium. Due to its ability to accurately measure cardiac functions, many reports are being published using this method. However, further development is anticipated in the future because consideration of various pathological conditions is still unclear, and although it is possible to measure by various ultrasound devices, it is only installed in expensive devices.

\section{REFERENCES}

1. Nakata M, Sakuma J, Takano M, et al. Assessment of fetal cardiac function with echocardiography. J Obstet Gynaecol Res 2020;46(1):31-38. DOI: 10.1111/jog.14143.

2. van Oostrum NHM, de Vet CM, van der Woude DAA, et al. Fetal strain and strain rate during pregnancy measured with speckle tracking echocardiography: a systematic review. Eur J Obstet Gynecol Reprod Biol 2020;250:178-187. DOI: 10.1016/j.ejogrb.2020.04.002.

3. Meister M, Axt-Fliedner R, Graupner $\mathrm{O}$, et al. Atrial and ventricular deformation analysis in normal fetal hearts using two-dimensional speckle tracking echocardiography. Fetal Diagn Ther 2020;47(9):699710. DOI: 10.1159/000508881.

4. Barker PC, Houle H, Li JS, et al. Global longitudinal cardiac strain and strain rate for assessment of fetal cardiac function: novel experience with velocity vector imaging. Echocardiography 2009;26(1):28-36. DOI: 10.1111/j.1540-8175.2008.00761.x.

5. Ishii T, McElhinney DB, Harrild DM, et al. Circumferential and longitudinal ventricular strain in the normal human fetus. J Am Soc Echocardiogr 2012;25(1):105-111. DOI: 10.1016/j.echo.2011.09.016.

6. Kapusta L, Mainzer G, Weiner Z, et al. Second trimester ultrasound: reference values for two-dimensional speckle tracking-derived longitudinal strain, strain rate and time to peak deformation of the fetal heart. J Am Soc Echocardiogr 2012;25(12):1333-1341. DOI: 10.1016/j.echo.2012.09.011.

7. Kapusta L, Mainzer G, Weiner Z, et al. Changes in fetal left and right ventricular strain mechanics during normal pregnancy. J Am Soc Echocardiogr 2013;26(10):1193-1200. DOI: 10.1016/j.echo.2013.06.007.

8. Matsui $H$, Germanakis I, Kulinskaya E, et al. Temporal and spatial performance of vector velocity imaging in the human fetal heart. Ultrasound Obstet Gynecol 2011;37(2):150-157. DOI: 10.1002/ uog.8815.

9. Peng QH, Zhou QC, Zeng S, et al. Evaluation of regional left ventricular longitudinal function in 151 normal fetuses using velocity vector imaging. Prenat Diagn 2009;29(12):1149-1155. DOI: 10.1002/pd.2386.

10. Pu DR, Zhou QC, Zhang $M$, et al. Assessment of regional right ventricular longitudinal functions in fetus using velocity vector imaging technology. Prenat Diagn 2010;30(11):1057-1063. DOI: 10.1002/pd.2611.

11. Dahlbäck C, Gudmundsson S. Increased pulsatility in the fetal ductus venosus is not related to altered cardiac strain in high-risk pregnancies. J Matern Fetal Neonatal Med 2016;29(8):1328-1333. DOI: 10.3109/14767058.2015.1047337.

12. van Oostrum NHM, van der Woude DAA, Clur SB, et al. Right ventricular dysfunction identified by abnormal strain values precedes evident growth restriction in small for gestational age fetuses. Prenat Diagn 2020;40(12):1525-1531. DOI: 10.1002/pd.5805.

13. Crispi F, Bijnens B, Figueras F, et al. Fetal growth restriction results in remodeled and less efficient hearts in children. Circulation 2010;121(22):2427-2436. DOI: 10.1161/CIRCULATIONAHA.110.937995.

14. van Oostrum NHM, Derks K, van der Woude DAA, et al. Twodimensional speckle tracking echocardiography in fetal growth restriction: a systematic review. Eur J Obstet Gynecol Reprod Biol 2020;254:87-94. DOI: 10.1016/j.ejogrb.2020.08.052. 
15. Wohlmuth C, Boudreaux D, Moise KJ, et al. Cardiac pathophysiology in twin-twin transfusion syndrome: new insights into its evolution. Ultrasound Obstet Gynecol 2018;51(3):341-348. DOI: 10.1002/ uog. 17480 .

16. Raboisson MJ, Fouron JC, Lamoureux J, et al. Early intertwin differences in myocardial performance during the twin-to-twin transfusion syndrome. Circulation 2004;110(19):3043-3048. DOI: 10.1161/01.CIR.0000146896.20317.59.

17. Taylor-Clarke MC, Matsui H, Roughton $\mathrm{M}$, et al. Ventricular strain changes in monochorionic twins with and without twin-to-twin transfusion syndrome. Am J Obstet Gynecol 2013;208(6):462.e1-6. DOI: 10.1016/j.ajog.2013.02.051.

18. Rychik J, Zeng S, Bebbington $M$, et al. Speckle tracking-derived myocardial tissue deformation imaging in twin-twin transfusion syndrome: differences in strain and strain rate between donor and recipient twins. Fetal Diagn Ther 2012;32(1-2):131-137. DOI: 10.1159/000335403.

19. Harbison AL, Pruetz JD, Ma S, et al. Evaluation of cardiac function in the recipient twin in successfully treated twin to twin transfusion syndrome (TTTS) using a novel fetal speckle tracking analysis. Prenat Diagn 2021;41(1):136-144. DOI: 10.1002/pd.5835.

20. Patey O, Carvalho JS, Thilaganathan B. Perinatal changes in fetal cardiac geometry and function in diabetic pregnancy at term.
Ultrasound Obstet Gynecol 2019;54(5):634-642. DOI: 10.1002/ uog.20187.

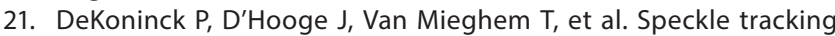
echocardiography in fetuses diagnosed with congenita diaphragmatic hernia. Prenat Diagn 2014;34(13):1262-1267. DOI: 10.1002/pd.4461.

22. Cohen J, Binka E, Woldu K, et al. Myocardial strain abnormalities in fetuses with pulmonary atresia and intact ventricular septum. Ultrasound Obstet Gynecol 2019;53(4):512-519. DOI: 10.1002/ uog.19183.

23. Nagasaki S, Nakata M, Takano M, et al. Feasibility of automated fetal fractional shortening measurement with two-dimensional tracking and construction of a reference range for normal fetuses. J Med Ultrason (2001) 2019;46(4):467-472. DOI: 10.1007/s10396-019-009426.

24. Nagasaki S, Nakata M, Takano M, et al. Measurement of fetal automated fractional shortening using two-dimensional tracking in multiple centers. J Med Ultrason (2001) 2021;48(1):83-90. DOI: 10.1007/s10396-020-01069-9.

25. DeVore GR, Polanco B, Satou G, et al. Two-dimensional speckle tracking of the fetal heart: a practical step-by-step approach for the fetal sonologist. JUltrasound Med 2016;35(8):1765-1781. DOI: 10.7863/ ultra.15.08060. 